\title{
Nalmefene relieves epidural morphine-caused pruritus following caesarean section: a randomized and placebo-controlled clinical trial
}

Chaochao Zhong ( $\square$ zhong249767626@163.com )

Affiliated Hospital of Nantong University

\section{Xingguo $\mathrm{Xu}$}

Affiliated Hospital of Nantong University

Xiang Zhu

Affiliated Hospital of Nantong University

Yibin Qin

Affiliated Hospital of Nantong University

Haihang Miao

Affiliated Hospital of Nantong University

Xinchong Huang

Affiliated Hospital of Nantong University

\section{Su Cao}

Affiliated Hospital of Nantong University

\section{Research article}

Keywords: Nalmefene, Epidural morphine, Pruritus, Caesarean delivery

Posted Date: December 16th, 2019

DOI: https://doi.org/10.21203/rs.2.17436/v2

License: (c) (i) This work is licensed under a Creative Commons Attribution 4.0 International License.

Read Full License 


\section{Abstract}

Background. Epidural morphine has an effective analgesic effect in cesarean section patients; however, a very common adverse effect caused by epidural morphine is pruritus, which is difficult to treat or prevent. Here, we aimed to investigate whether a $\mu$-opioid antagonist with central and peripheral effects reduces morphine-induced pruritus.

Methods. In this prospective randomized trial, eighty patients scheduled for an elective cesarean section under spinal aesthesia with $3 \mathrm{mg}$ of epidural morphine were assigned into the nalmefene group $(n=40)$ or placebo group $(n=40)$. After delivery, either $50 \mu \mathrm{g}$ of intravenous nalmefene hydrochloride (Nalmefene group) or an equivalent amount of normal saline (Placebo group) was administered to the patients. In the meantime, an assessment of a series of side effects such as pruritus, nausea, and pain was conducted at $2,4,8,12$, and $24 \mathrm{~h}$ after epidural morphine administration.

Results. All eighty participants completed this trial. The total incidence of pruritus in the first 24 hours following the section was reduced in IV nalmefene group compared with the placebo group $(37.5 \% \mathrm{vs}$ $65 \%, P=0.003)$. Moreover, IV nalmefene administration relieved the pruritus intensity, whereas the difference in the incidence of nausea and vomiting between the two groups was not significant. Besides, the nalmefene group displayed significantly higher pain scores at 8, 12 and $24 \mathrm{~h}$ than the placebo group (all $\mathrm{P}<0.05)$. However, no significant difference in the percentage of patients with an analgesic treatment was found between the two groups $(P=0.37)$.

Conclusion. In this study, a single dose of $50 \mu \mathrm{g}$ of IV nalmefene was found to decrease the overall severity and incidence of epidural morphine-induced pruritus, but cause no adverse effect on postoperative analgesia.

\section{Background}

Pruritus is one of the commonest adverse effects resulting from use of epidural morphine for postoperative analgesia following cesarean section; it reportedly occurs in $30-100 \%$ of the patients ${ }^{1}$. Although pruritus does not impose a remarkable influence on the patient's health ${ }^{2}$, it causes a disagreeable sensation that elicits a scratching reflex. At present, epidural morphine is still being used in the clinic due to its excellent postoperative analgesia after caesarean delivery. ${ }^{1}$

In fact, many medications have been studied for preventing epidural opioid-associated pruritus, and morphine-induced pruritus showed no or weak response to antihistamines, serotonin antagonists, and propofol ${ }^{3,4}$. More investigations have been done on several other drugs with different effects as well as side effects, including gabapentin, nalbuphine and pentazocine. Currently, the precise mechanism underlying neuraxial opioid-caused pruritus remains unknown. As opioid receptors are highly expressed in the central nervous system ${ }^{5}$, it is generally believed that $\mu$ receptors mediate the above pruritus. Therefore, naloxone, a $\mu$-opioid antagonist, can be the most effective therapeutic drug for epidural 
morphine-caused pruritus. However, the use of $\mu$-opioid antagonists may potentially cause an adverse impact on analgesia ${ }^{3}$.

Experimental evidence suggests that naloxone alleviates the itching of animals caused by opioids ${ }^{6}$; however, it can antagonize the analgesic effect while relieving itching. Nalmefene is a derivative of naloxone, which displays higher affinity with $\mu$ receptor as well as a long-lasting effect. Studies have shown that low doses of nalmefene have no effect on analgesia or postoperative pain ${ }^{7}$. To date, there are few studies focused on the treatment of pruritus with nalmefene. Here, we hypothesized that IV nalmefene may effectively relieve epidural morphine-caused pruritus following caesarean section.

\section{Methods}

This study was registered on April 2, 2019, and was assigned a Chinese Clinical Trial Registry number: ChiCTR1900022268

(http://www.chictr.org.cn/edit.aspx). This work has been

reported in accordance with the guidelines of the Consolidated Standards of Reporting Trials (CONSORT).

In this study, a randomized and placebo-controlled trial was performed on a total of 80 patients undergoing elective cesarean section from April of 2019 to June of 2019.

We recruited the following individuals in the study: ASA class $\$ or》 women aged 18 years or older who were scheduled for an elective caesarean section under epidural anaesthesia. The following exclusion criteria were applied in this trial: no consent obtained for this study, coagulation dieases, liver or kidney failure, pre-existing itching, a history of lumbar spine surgery.

To perform epidural anesthesia, an 18-gauge epidural needle was used and an epidural catheter was inserted at the T12-L1 or L1-L2 level of the patients. $60 \mathrm{mg}(12 \mathrm{ml})$ of $0.5 \%$ ropicacaine and $3 \mathrm{mg}$ of morphine were then administered respectively. The patients were maintained at supine position, and maintenance of a systolic blood pressure of at least $100 \mathrm{mmHg}$ throughout the operation was performed by using colloid liquid as well as intermittent injection of phenylephrine.

All patients were randomly assigned into the placebo group (injection with saline) and nalmefene group (injection with 50 ug of nalmefene). Nalmefene or placebo was administered just prior to the end of surgery, and intraoperative anaesthesia and postoperative care were routinely conducted. Baseline data of the patients, including age, characteristics, and operation time, were collected. The rate of pain or nausea/vomiting during the operation was recorded. Following the operation, the severity of itching, pain scores, and other side effects such as nausea and vomiting were evaluated $2,4,8,12$, and $24 \mathrm{~h}$ after administration of nalmefene or saline, respectively. At 24 hours following delivery, patients were reexamined to determine the total incidence of pruritus, regardless of the requirement for a treatment of pruritus or pain. The assessment of pruritus intensity was performed based on verbal rating scale ( 0 no 
pruritus, 1 mild pruritus, 2 moderate pruritus, 3 severe pruritus). ${ }^{3}$ VAS scores for pain ranged from 0 (no symptom) to 10 (unbearable pain). $4 \mathrm{mg}$ of Intravenous ondansteron was administered for relieving nausea and vomiting. Non-steroidal anti-inflmmatory drugs or intravenous morphine were used to assist postoperative analgesia when necessary.

\section{Statistics}

The software SPSS 13.0 was used for statistical analysis in this study. The sample size was calculated by using Power Analysis and Sample Size (PASS) software (NCSS) (LLC, UT, USA) with $a=0.05$ and $\beta=0.95$. Clinically, a reduction in the incidence of pruritus by $30 \%$ indicates a therapeutic significance. The preliminary analysis revealed a high incidence of pruritus (65\%) in the control group. Thus, each of the groups in this study must contain no less than 32 patients in order to fulfill the requirement for a $30 \%$ reduction in the incidence of pruritus.

Levene method was used for testing normality. The normally distributed data was presented as the mean and standard deviation (SD), while the other data was presented as the mean and interquartile range. Count data groups were analyzed using chi-square test. Comparative analysis of proportional data was carried out based on the $\chi 2$ test as well as Fisher's exact test. Nemenyi test was performed for determining the difference between the two groups. Statistically, $\mathrm{P} \llbracket 0.05$ represented a significant difference.

\section{Results}

80 out of 84 initially enrolled patients participated in this study; all these patients were assigned into the placebo group $(n=40)$ or the nalmefene group $(n=40)$. As indicated in Table 1 , these two groups displayed no difference in the baseline characteristics.

As summarized in Table 4, based on the primary outcome measures, the incidence of pruritus in the nalmefene group and the placebo group was $32.5 \%(13 / 40)$ and $65 \%(26 / 40)$, respectively. The difference in the incidence between the two groups was significant $(P<0.05)$. Notably, while 4 patients in the nalmefene group received treatment of pruritus with ondansetron or antihistamine, 19 patients in the placebo group needed to be treated for pruritus (Table $4, P<0.01$ ). Moreover, the two groups exhibited significant differences in the severity of pruritus $2,4,8$, and $12 \mathrm{~h}$ after the administration of epidural morphine, respectively ( Table $2, \mathrm{P}<0.05$ ).

As shown in Table 3, the two groups had similar VAS scores at 2, 4, and $24 \mathrm{~h}$ after the administration, whereas significantly higher VAS scores at 8 and $12 \mathrm{~h}$ were present in the nalmefene group compared with the placebo group. Clearly, there was no significant difference between the two groups in the number of patients receiving treatment for pain ( Table $4,10 \%$ vs $15 \%, p=0.37$ ).

The status of nausea and vomiting within the first $24 \mathrm{~h}$ following the operation was analyzed in all patients in Table 4. The incidence of nsusea in the placebo and nalmefene groups was $17.5 \%$ and $25 \%$, 
respectively, while the incidence of vomiting was $7.5 \%$ vs $10 \%$ between the two groups. Statistically, no significant differences in the above two symptoms were found between the two groups (nsusea, $P=0.29$; vomiting, $\mathrm{P}=0.51)$.

Fig. 1 The screening, exclusion and randomization for all patients in this study were illustrated in the chart.

Table 1. Comparative analysis of the characteristics, obstetric, and baseline clinical data of the patients between the two groups

\begin{tabular}{lcc}
\hline & Placebo $(\mathrm{n}=40)$ & Nalmefene $(\mathrm{n}=40)$ \\
\hline Age $(\mathrm{y})$ & $27.5 \pm 3.7$ & $26.4 \pm 3.6$ \\
Weight $(\mathrm{kg})$ & $73.6 \pm 4.1$ & $73.4 \pm 4.6$ \\
BMI $(\mathrm{kg} / \mathrm{m} 2)$ & $29.9 \pm 2.7$ & $29.9 \pm 2.8$ \\
Nulliparous & $25(62.5 \%)$ & $23(57.5 \%)$ \\
Op time (min) & $46.6 \pm 4.6$ & $45.5 \pm 4.2$ \\
\hline
\end{tabular}

Table 2. The assessment of the clinical manifestation of pruritus at $2,4,8,12$, and $24 \mathrm{~h}$ following epidural injection of morphine in all patients of the two groups

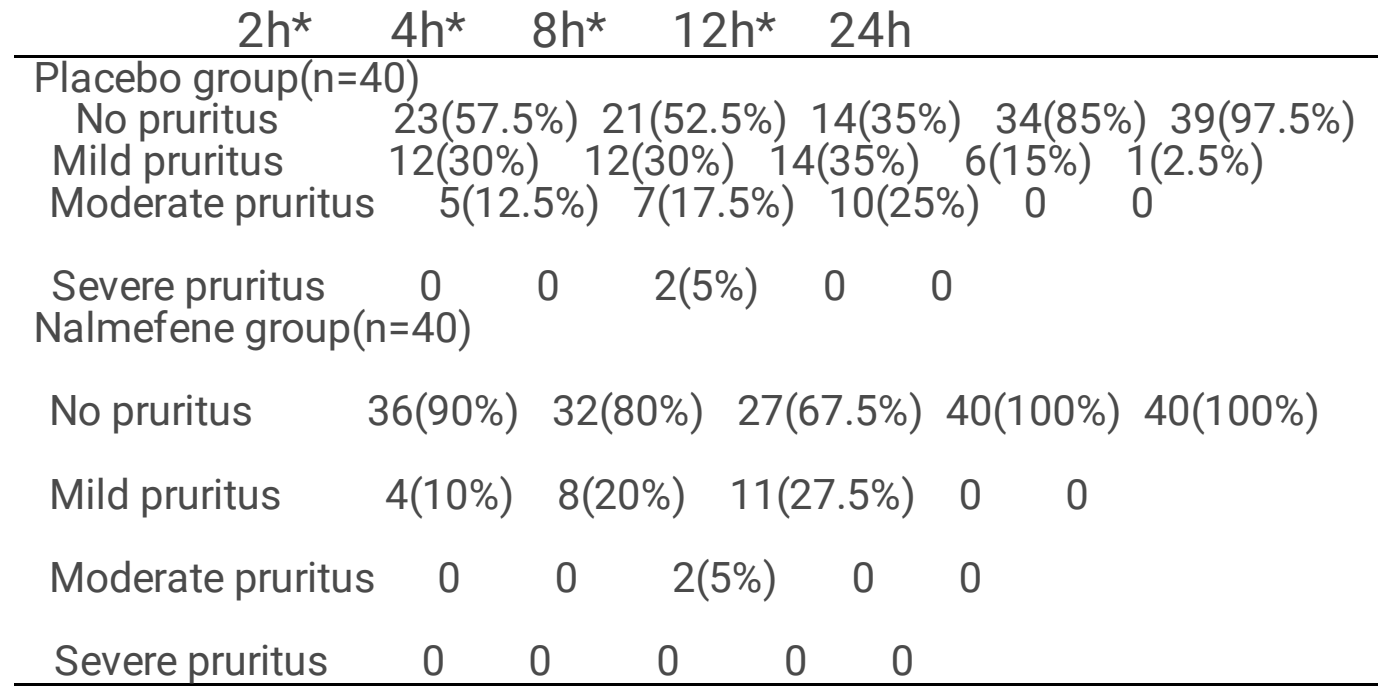

Table 3. Comparative analysis of the pain VAS scores between the two groups 


\begin{tabular}{ccccccc}
\hline $2 \mathrm{~h}$ & $4 \mathrm{~h}$ & $8 \mathrm{~h}^{*}$ & $12 \mathrm{~h} *$ & $24 \mathrm{~h}$ & & \\
\hline Placebo group & $1.2 \pm 0.8$ & $1.9 \pm 0.8$ & $2.1 \pm 0.9$ & $2.2 \pm 0.7$ & $2.0 \pm 0.8$ \\
Nalmefene group & $1.2 \pm 0.7$ & $1.9 \pm 1.1$ & $2.8 \pm 0.8$ & $3.1 \pm 0.7$ & $2.9 \pm 0.8$ \\
\hline
\end{tabular}

Table 4. Comparative analysis of the adverse events in the two groups

\begin{tabular}{lcccc}
\hline \multicolumn{4}{c}{ Placebo $(\mathrm{n}=40)$} & \multicolumn{3}{c}{ Nalmefene $(\mathrm{n}=40)$} & $P$ \\
\hline Nausea(n) & 7 & 10 & 0.29 & \\
Vomitting(n) & 3 & 4 & 0.51 & \\
Pruritus(n) & 26 & 13 & $<0.05$ & \\
treatment of pruritus(n) & 19 & 4 & $<0.01$ \\
treatment of pain $(\mathrm{n})$ & 4 & 6 & 0.37 \\
\hline
\end{tabular}

\section{Discussion}

In this prospective, randomized, and placebo-controlled clinical trial, a comparative analysis was performed on the effects of nalmefene and normal saline on pruritus relief in post-cesarean section patients. Here, we showed that a single dose of $50 \mu \mathrm{g}$ of IV nalmefene reduced the overall severity and incidence of epidural morphine-induced pruritus, but caused no adverse effect on the postoperative analgesia.

Pregnant women are more prone to itching that occurs in the face, neck, and upper thorax; the incidence of itching among these women is dose dependent ${ }^{8,910}$. While the precise mechanisms of pruritus is still not clear, a lot of progress has recently been made in identifying signal molecules as potential targets for anti-pruritus therapies ${ }^{11,12}$. As $\mu$-opioid receptor antagonists can effectively relieve pruritus by acting on central and probably peripheral targets ${ }^{13}$, the interactions of opioid with $\mu$-opioid receptors may mediate opioid-induced pruritus. With the increased knowledge, we can identify more therapeutic targets and then improve the efficacy of the pruritus treatments as well as reducing the adverse effects. As an $\mu$ receptor antagonist, naloxone has been shown to be the most effective therapeutic drug for pruritus caused by epidural morphine. However, only a small dosage of naloxone can be applied for relieving pruritus following caesarean delivery, due to the fact that a high dosage of naloxone antagonizes the analgesic action of opioids ${ }^{14}$. As a novel $\mu$-opioid antagonist derived from naloxone, nalmefene has a stronger affinity with $\mu$-opioid compared with naloxone. While both naloxone and nalmefene can easily cross the blood-brain barrier ${ }^{15}$, naloxone is metabolized faster in the body. Importantly, the clinically used dose of nalmefene appears to antagonize the side effects of opioids, but cause little effect on the pain. 
In this clinical trial, we analyzed the effect of nalmefene on epidural morphine-caused pruritus, and found that nalmefene administration decreased the incidence of pruritus to $32.5 \%$. Compared with the previous reports ${ }^{16,17}$, a higher percentage of pruritus was observed in this study. The above discrepancy may be attributed to the varied dosage of morphine used in the studies. Besides, the higher rate of pruritus observed in this trial may result from the improper dosage and administration time, or the fact that nalmefene does not affect other itching transmitters, such as prostaglandins ${ }^{18}$, the neurotransmitters glutamate and $\mathrm{GABA}^{19}$, and NMDA receptors ${ }^{20}$.

In the present study, we observed that while the two groups had similar VAS scores at 2, 4, and $24 \mathrm{~h}$ after epidural injection of morphine, slightly higher VAS scores at 8 and $12 \mathrm{~h}$ were evident in the nalmefene group compared with the placebo group. This observation suggested that nalmefene reduced pruritus and impaired the analgesic efficacy of epidural morphine. Surprisingly, no discrepancy in the percentage of patients receiving analgesic treatment was detected between the two groups, suggesting that nelmefene reduces the overall severity and incidence of epidural morphine-caused pruritus, but has no adverse effect on postoperative analgesia. In addition to itching, epidural morphine causes nausea as well as vomiting due to the activation of serotonin receptors in the trigger area of the vomiting chemoreceptor $^{21}$. In the study, we showed that the difference in both nausea and vomiting was not significant between the two groups.

There are some limitations in this clinical trial. First, we only analyzed the effect of a single dose of nalmefene on relieving epidural morphine-caused pruritus in patients following Caesarean section. Therefore, a change in the dosage of nalmefene or the type of patients may yield different results. Secondly, testing a dose-dependent effect of nalmefene on pruritus is still lacking in the study. Thus, future studies will determine whether the anti-pruritus effect of nalmefene is dose-dependent. If it was the case, we need to further identify the optimal dose of nalmefene that can be applied for relieving pruritus as well as reducing the side effects on analgesia to the greatest extent. Moreover, it would be important to investigate whether nalmefene also displays a therapeutic effect on pruritus caused by other opioids with epidural administration.

\section{Conclusions}

This study revealed that a single dose of $50 \mu \mathrm{g}$ of IV nalmefene decreases the overall severity and incidence of epidural morphine-caused pruritus, but causes no adverse effect on postoperative analgesia.

\section{Abbreviations}

ASA: American society of anesthesiology; VAS: visual analog scale

PASS: Power Analysis and Sample Size

\section{Declarations}


Ethics approval and consent to participate

This study was approved by the Ethics Committee of Hospital Affiliated with Nantong University (approved number: 2018-K067). The written informed consent was obtained from each of the participants.

Consent for publication

We acknowledge that upon the publication, the ownership of copyright for this work will be transferred to the journal "BMC anesthesiology". We declare that this work was original research, and all authors participated in drafting, revising, and approving the submitted manuscript.

Availability of data and materials

We presented all necessary supporting data in the paper. Upon request, the datasets used in this study can be available from the corresponding author.

Competing interests

The authors declare that no competing interests exist in the paper.

Funding

This study was supported by a grant from the Science and Technology Bureau of Nantong municipality.

Authors' contributions

CCZ performed the clinical trials, and wrote the manuscript. XGX $\square X Z$ and HHM conducted the statistical analysis as well as working in the data collection. YBQ and SC worked in revising the manuscript. Each of the authors read the manuscript and approved the final version.

Acknowlegements

We thank Su Cao from department of anesthesiology in Nantong University Hospital for her supervision in this project.

Statement

The study adheres to CONSORT guidelines.

Authors' Information

Chaochao Zhong, Email: zhong249767626@163.com

Xingguo Xu, Email: TDFYXXG@126.com 
Xiang Zhu, Email: Bobofly8850@sina.com

YiBin Qin, Email: 572537172@qq.com

Haihang Miao, Email: miaohaihang@163.com

Xinchong Huang, Email: hxcnt115@sohu.com

Su Cao, Email: mzkcs@sina.com

\section{References}

1. Kumar K, Singh SI. Neuraxial opioid-induced pruritus: an update. J Anaesthesiol Clin Pharmacol 2013;29:303-7.

2. Yosipovitch G, Carstens E, McGlone F. Chronic itch and chronic pain: analogous mechanisms. Pain 2007;131:4-7.

3. Lockington PF, Fa'aea P. Subcutaneous naloxone for the prevention of epidural morphine induced pruritus in elective caesarean delivery. Anesthesia 2007;62:672-676.

4. Bonnet MP, Marret E, Josserand J. Effect of prophylactic 5-HT3 receptor antagonists on pruritus induced by neuraxial opioids: a quantitative systematic review. Br J Anaesth 2008; 101:301-9.

5. Waxler B, Dadabhoy Z, Stojiljkovic L. Primer of postoperative pruritus for anesthesiologists. Anesthesiology 2005; 103:168-178.

6. Sakakihara M, Imamachi N, Saito Y. Effects of epidural k-opioid receptor agonist on morphineinduced itch and antinociception in mice. Reg Anesth Pain Med 2016;41:69-74.

7. Miller JL, Hagemann TM. Use of pure opioid antagonists for management of opioid-induced pruritus. Am J Health Sys Pharm 2011;68:1419-25.

8. Yeh HM, Chen LK, Lin CJ et al. Prophylactic intravenous ondansetron reduces the incidence of epidural morphine-induced pruritus inpatients undergoing cesarean delivery. Anesth Analg 2000;91:172-175.

9. Horta ML, Ramos L, Goncalves ZR. The inhibition of epidural morphine-induced pruritus by epidural droperidol. Anesth Analg. 2000;90:638-641.

10. Szarvas S, Harmon D, Murphy D. Neuraxial opioid-induced pruritus: a review. J Clin Anesth 2003;15:234-239.

11. Bautista DM, Wilson SR, Hoon MA. Why we scratch an itch: the molecules cells and circuits of itch. Nat. Neurosci 2014;17:175-182.

12. Barry DM, Munanairi A, Chen ZF. Spinal mechanisms of itch transmission. Neurosci Bull. 2018;34:156-164.

13. Papoiu ADP, Kraft RA, Coghill RC et al. Butorphanol suppression of histamine itch is mediated by nucleus accumbens and septal nuclei: a pharmaco-logical fMRI study. J Invest Dermatol 
$2015 ; 135: 560-568$.

14. Kelly MC, Carabine UA, Mirakhur RK. Epidural diamorphine for analgesia after caesarean section. A dose finding study and assessment of side-effects. Anesthesia. 1998;53:231-237.

15. Viscusi ER, Gan TJ, Leslie JB, et al. Peripherally acting mu-opioid receptor antagonists and postoperative ileus: mechanisms of action and clinical applicability. Anesth Analg 2009; 108:181122.

16. Girgin NK, Gurbet A, Turker G, et al. Epidural morphine in anesthesia for combinations of low-dose epidural morphine and spinal bupivacaine. J Clin Anesth 2008;20:180-185.

17. Sarvela J, Halonen P, Soikkeli A, et al. A double-blinded, randomized comparison of epidural and epidural morphine for elective cesarean delivery. Anesth Analg 2002;95:436-440.

18. Colbert S, O'Hanlon DM, Galvin S, et al. The effect of rectal diclofenac on pruritus in patients receiving epidural morphine. Anesthesia 1999;54:948-952.

19. Ballantyne JC, Loach AB, Carr DB. Itching after epidural and spinal opiates. Pain 1998;33:149-160.

20. Jinks SL, Carstens E. Spinal NMDA receptor involvement in eapansion of dorsal horn neuronal receptive field area produced by intracutaneous histamine. J Neurophysiol 1998;79:1613-1618.

21. Chaney MA. Side effects of epidural and epidural opioids. Can J Anaesth. 1995;42:891-903.

\section{Figures}




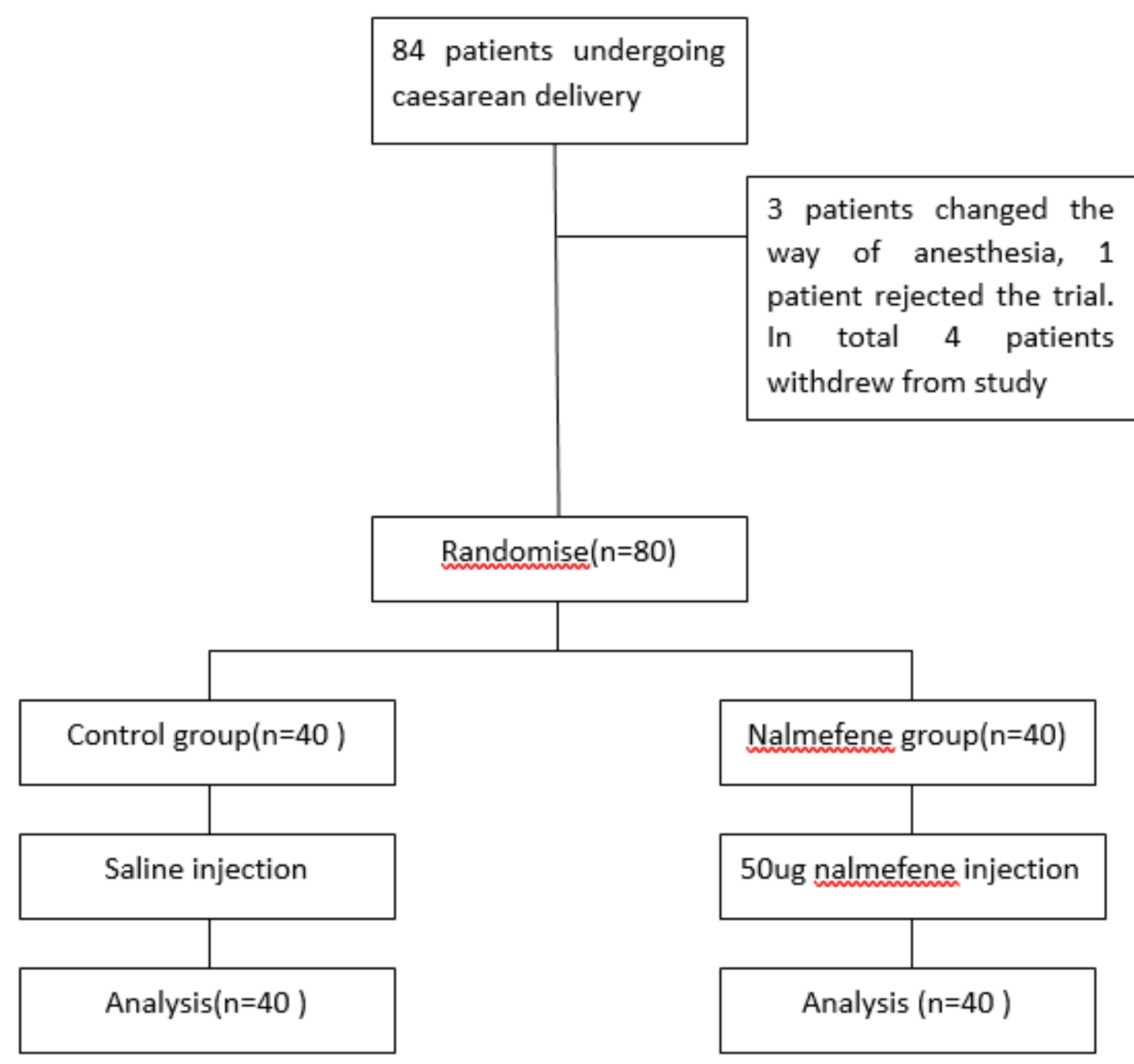

Figure 1

patient enrollment flow diagram. This illustrates the flow of all patients screened, excluded, and randomized

\section{Supplementary Files}

This is a list of supplementary files associated with this preprint. Click to download.

- CONSORTChecklist.doc 